\title{
Evaluation of pembrolizumab for the treatment of advanced non-small cell lung cancer: a retrospective, single-centre, single-arm study
}

\author{
Tianxing Guo ${ }^{1 \#}$, Yun Ding ${ }^{2 \#}$, Long Chen ${ }^{2}$, Lihuan Zhu ${ }^{1}$, Jinlan Lin ${ }^{3}$, Jiguang Zhang ${ }^{1}$, Yangyun Huang ${ }^{1}$, \\ Wujin Li ${ }^{1}$, Rongjia Lin ${ }^{1}$, Xiaojie Pan ${ }^{1}$ \\ ${ }^{1}$ Department of Thoracic Surgery, Shengli Clinical Medical College of Fujian Medical University, Fujian Provincial Hospital, Fuzhou, China; \\ ${ }^{2}$ Shengli Clinical Medical College of Fujian Medical University, Fuzhou, China; ${ }^{3}$ Department of Thoracic Oncology, Fujian Cancer Hospital, Fujian \\ Medical University Cancer Hospital, Fuzhou, China \\ Contributions: (I) Conception and design: T Guo, Y Ding; (II) Administrative support: X Pan; (III) Provision of study materials or patients: T Guo, \\ J Lin, J Zhang; (IV) Collection and assembly of data: Y Ding, L Zhu, Y Huang, W Li; (V) Data analysis and interpretation: L Chen, R Lin; (VI) \\ Manuscript writing: All authors; (VII) Final approval of manuscript: All authors. \\ \#These authors contributed equally to this work. \\ Correspondence to: Xiaojie Pan. Department of Thoracic Surgery, Shengli Clinical Medical College of Fujian Medical University, Fujian Provincial \\ Hospital, 134 East Street, Fuzhou, China. Email: pxj1028@hotmail.com.
}

Background: Immune checkpoint inhibitors (ICIs) provided a paradigm shift for advanced non-small cell lung cancer (NSCLC) treatment and improved the clinical prognosis of such patients. Pembrolizumab is a humanized anti-programmed death cell protein 1 (PD-1) monoclonal antibody, approved for the treatment of patients with advanced or metastatic NSCLC. This article investigated and reported on the efficacy and safety of pembrolizumab in the treatment of advanced NSCLC in our center since 2019.

Methods: Patients with clinical stage III-IV NSCLC treated with pembrolizumab for $\geq 4$ cycles were enrolled as participants in this study. Pembrolizumab was administered intravenously at a dose of $2 \mathrm{mg} / \mathrm{kg}$ every 3 weeks. A cycle was defined as 3 weeks of treatment. We assessed the efficacy and safety of pembrolizumab through the collection of researcher-assessed tumor response, survival, and safety data.

Results: A total of 24 patients were included in this study. The median follow-up time was 9 months (3-20 months) and the median period of pembrolizumab therapy was 7 cycles (4-21 cycles). The objective response rate (ORR) was $45.8 \%$ and disease control rate (DCR) was $70.8 \%$. The median overall survival (OS) and progression-free survival (PFS) times were not reached. A total of 2 programmed death-ligand 1 (PDL1)-negative participants were treated with pembrolizumab combined with chemotherapy and there was no significant progression during the follow-up period. During the follow-up period, 8 patients underwent surgery. The major pathological response (MPR) was $75 \%$ and pathological complete response (pCR) was $50 \%$. A case that was preoperatively diagnosed with clinical stage IV achieved pCR after 6 cycles of pembrolizumab combined with chemotherapy. The incidence of adverse effects (AEs) was 83.3\%, and 16.7\% of these were serious AEs (grade $\geq 3$ ), which was similar to the incidence reported in previous studies.

Conclusions: This real-world data supports the use of pembrolizumab for advanced NSCLC, including those cases that are PD-L1 negative. More importantly, pembrolizumab immunotherapy can also provide the potential of local treatment for patients with advanced NSCLC, which has wide application prospects in the field of surgery.

Keywords: Pembrolizumab; immunotherapy; advanced non-small cell lung cancer (NSCLC); efficacy; safety

Submitted Oct 30, 2020. Accepted for publication Jan 02, 2021.

doi: $10.21037 /$ jtd-20-3413

View this article at: http://dx.doi.org/10.21037/jtd-20-3413 


\section{Introduction}

Lung cancer is the most common cause of cancer-related death, for which non-small cell lung cancer (NSCLC) is the most prevalent type of lung cancer (1). As its symptoms are non-specific at the early stage, the majority of the patients are diagnosed at an advanced stage, which generally indicates a poor outcome (2). Recently, the rise of immune checkpoint inhibitors (ICIs) has expedited the development of treatment for advanced NSCLC and ICIs have been applied to treat various types of malignant tumors. Programmed death cell protein $1(\mathrm{PD}-1)$ is a novel member of the immunoglobulin gene superfamily, with notably high expression on T cells. Programmed death-ligand 1 (PD-L1) is one of the primary ligands of PD-1 and is expressed on many types of tumor cells and antigen presenting cells. Tumor-associated PD-L1 ligates to PD-1 on the surface of $\mathrm{T}$ cells, thereby inhibiting the function of $\mathrm{T}$ cells, which leads to suppression of their killing effect on tumor cells (3). The ICIs targeting PD-1/PD-L1 can prevent PD-1 and PD-L1 from binding and permit the T-cell-mediated death of tumor cells. In recent years, immunotherapy based on inhibition of PD-1/PD-L1 has achieved encouraging therapeutic effects in advanced NSCLC. However, there are discrepancies regarding the efficacy and safety of immunotherapy reported by different agencies among different populations.

Pembrolizumab is a humanized monoclonal immunoglobulin IgG4 antibody targeted against PD-1, which blocks the interaction of PD-1 with its ligand PDL1. Moreover, pembrolizumab has been approved as a first-line treatment for advanced NSCLC patients with a PD-L1 tumor proportion score (TPS) $\geq 50 \%(4,5)$. The KEYNOTE-024 Phase III trial further characterized the safety and efficacy of pembrolizumab monotherapy for advanced NSCLC patients with PD-L1 TPS $\geq 1 \%$ (6). In addition, the KEYNOTE-189 and KEYNOTE-407 trials $(7,8)$ showed that, for patients with epidermal growth factor receptor gene (EGFR)/anaplastic lymphoma kinase (ALK) negative metastatic NSCLC, pembrolizumab plus chemotherapy led to improved patient outcomes compared to single-agent chemotherapy. These studies suggest pembrolizumab has a good curative treatment effect for advanced-stage NSCLC, either as a monotherapy or in combination with chemotherapy.

In our center, we have used pembrolizumab to treat several stage III-IV NSCLC patients and have achieved good results. Eight of them underwent surgery after pembrolizumab therapy and the pathological complete response (pCR) was $50 \%$, which is unprecedented. This article reports on the efficacy and safety of pembrolizumab therapy for advanced NSCLC in the Fujian Provincial Hospital since January 2019. We present the following article in accordance with the STROBE reporting checklist (available at http://dx.doi.org/10.21037/jtd-20-3413).

\section{Methods}

\section{Participants and treatments}

We collected the clinical data of stage III-IV NSCLC patients in the Fujian Provincial Hospital from January 2019 to September 2020. The inclusion criteria were as follows: (I) diagnosed by pathology or cytology; (II) clinical stage III or stage IV NSCLC according to the 8th edition of the Union for International Cancer Control/American Joint Committee on Cancer tumor, node, metastasis (UICC/AJCC TNM) staging system (9); (III) received $\geq 4$ cycles of pembrolizumab therapy; (IV) performance status (PS) scores of 0-2 according to the Eastern Cooperative Oncology Group (ECOG) before pembrolizumab therapy. The exclusion criteria were as follows: (I) NSCLC combined with other tumors; (II) response could not be evaluated after pembrolizumab therapy. According to the above standards, a total of 24 participants were included in the study. Pembrolizumab was administered intravenously at a dose of $2 \mathrm{mg} / \mathrm{kg}$ every 3 weeks. A cycle was defined as 3 weeks of treatment. Treatment was discontinued in the event of disease progression, unacceptable toxicity, or participant request to discontinue. This study was conducted in accordance with the declaration of Helsinki (as revise in 2013). The study was approved by the Institutional Research Ethics Board of the Fujian Provincial Hospital Ethics Committee (No.: 2020-015-01). Individual consent for this retrospective analysis was waived.

\section{Clinicopathological characteristics}

The medical records of all participants were reviewed retrospectively and clinical characteristics were gathered (age, gender, smoking history, tumor histology, tumor metastasis status, clinical staging, ECOG score, gene status, expression of PD-L1, and previous treatment line). To determine the expression of PD-L1, hematoxylin and eosin (HE) staining of the pathological sections of tumors or metastatic lymph nodes was performed. PD-L1 was 
evaluated through immunohistochemistry staining using 22C3 (DAKO, Carpinteria, CA, USA) or SP142 (Burning Rock Biotech, Guangzhou, China) anti-PD-L1 antibody. The percentage of tumor cells positive for PD-L1 was performed using the TPS (10), which is the percentage of tumor cells showing partial or complete membrane staining $(\geq 1+)$ to all tumor cells present in the sample. The TPS $(\%)=$ numbers of tumor cells positive for PD-L1/numbers of living tumor cells $\times 100 \%$; PD-L1 negative, TPS $<1 \%$; PD-L1 weakly positive, TPS 1 to $49 \%$; PD-L1 highly expressed, TPS $\geq 50 \%$.

\section{Follow-up protocol and efficacy assessment}

All participants were followed up by collection of electronic medical records or telephone interview. The followup data included clinicopathological characteristics, treatment, disease progression, and survival. Baseline assessment was performed before initial pembrolizumab therapy and response evaluation was performed by computed tomography (CT) scan following every 2 cycles of treatment. Therapeutic response was defined according to the immune-related Response Evaluation Criteria in Solid Tumors (irRECIST) (11). Response was categorized as complete response (CR), partial response $(\mathrm{PR})$, stable disease (SD), and progressive disease (PD). For participants undergoing surgery, we also calculated pCR and major pathological response (MPR). Additional efficacy parameters included objective response rate (ORR), disease control rate (DCR), progression free survival (PFS) and overall survival (OS). The ORR was defined as the CR plus PR rates. The DCR was defined as the CR plus PR plus SD rates. Calculation of PFS was from the start date of pembrolizumab therapy to the date of disease progression or death. Definition of OS was the time from the first dose of pembrolizumab to death from any cause. Efficacy assessments were made at least every 2 cycles. The deadline for follow-up was August 31, 2020, and the median followup time was 9 months. All 24 participants were alive and no patients were lost to follow-up at the deadline.

\section{Safety assessment}

All adverse events (AEs) were graded according to the National Cancer Institute Common Terminology Criteria for Adverse Events (NCI CTCAE) version 5.0 (12). During every medical visit, routine physical and laboratory examinations were performed for all participates.
Laboratory examinations included hematology and biochemical panels and thyroid function tests. Major AEs observed during the course of study were recorded.

\section{Statistical analysis}

Enumeration data are presented as number (n) and percentage (\%). The Kaplan-Meier method was used for survival analysis. The statistical software SPSS version 23.0 (SPSS Inc., IBM, Chicago, IL, USA) was used for statistical analysis. Kaplan-Meier survival curves were generated and analyzed using GraphPad Prism version 8.0 (GraphPad Software Inc., La Jolla, CA, USA).

\section{Results}

\section{Baseline characteristics of all participants}

A total of 24 patients were included $(17$ males and 7 females) with a median age of 59.0 years (47-75 years). The baseline characteristics of 24 patients are shown in Table 1.

Table 1 Clinicopathological characteristics of patients $(n=24)$

\begin{tabular}{lc}
\hline Characteristic & Value \\
\hline Period of pembrolizumab therapy, cycles & $7[4-21]$ \\
Median [range] & \\
Age, years & $59[47-75]$ \\
Median [range] & \\
Gender, $\mathrm{n}(\%)$ & $17(70.8)$ \\
Male & $7(29.2)$ \\
Female & \\
Smoking history, $\mathrm{n}(\%)$ & $14(58.3)$ \\
Yes & $10(41.7)$ \\
No & \\
ECoG PS, $\mathrm{n}(\%)$ & $9(37.5)$ \\
0 & $13(54.2)$ \\
1 & $2(8.3)$ \\
2 & $14(58.3)$ \\
Histology, $\mathrm{n}(\%)$ & $8(33.3)$ \\
\hline Adenocarcinoma & \\
\hline
\end{tabular}

Table 1 (continued) 
Table 1 (continued)

\begin{tabular}{lc}
\hline Characteristic & Value \\
\hline Large cell lung cancer & $2(8.3)$ \\
Clinical stage, $\mathrm{n}(\%)$ & \\
IIIA & $6(23.5)$ \\
IIIB & $8(23.5)$ \\
IV & $10(52.9)$ \\
Metastasis site, $\mathrm{n}(\%)$ & \\
Bone & $3(12.5)$ \\
Adrenal glands & $3(12.5)$ \\
Liver & $2(8.3)$ \\
Brain & $1(4.2)$ \\
PD-L1 expression status, $\mathrm{n}(\%)$ & \\
PD-L1 positive & $13(52.9)$ \\
PD-L1 negative & $2(11.8)$ \\
Unknown & $9(35.3)$ \\
Driver mutation, $\mathrm{n}(\%)$ & $18(75.0)$ \\
KRAS mutation & \\
BRAF mutation & \\
Pan-negative & \\
\hline Yembro & \\
\hline
\end{tabular}

ECOG, Eastern Cooperative Oncology Group; PD-L1, programmed death-ligand 1; KRAS, Kirsten rat sarcoma viral oncogene homolog; BRAF, v-raf murine sarcoma viral oncogene homolog B1.

A total of 14 cases had a history of smoking and 10 were non-smokers. The pathological type of 14 participants were adenocarcinoma, 8 were squamous carcinoma, and 2 were large cell cancer. There were 2 participants with an ECOG score of 0, 13 with an ECOG score of 1, and 9 with an ECOG score of 2. Clinical stage IIIA was diagnosed in 6 cases, stage IIIB in 8 cases, and stage IV in 10 cases. Distant metastasis was observed in 7 cases (3 in bone, 3 in adrenal glands, 2 in liver, and 1 with brain metastases). The

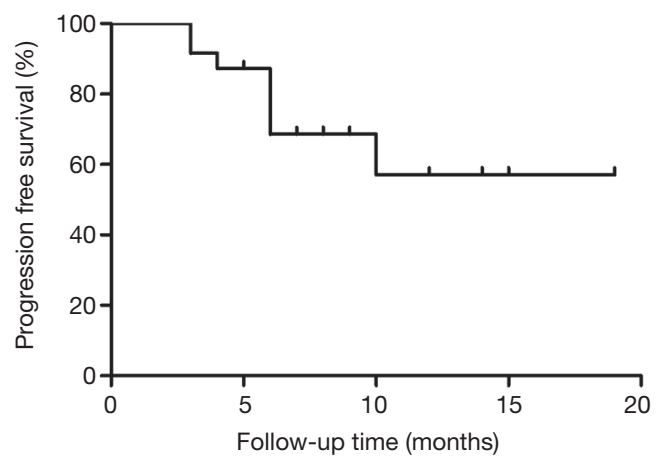

Figure 1 PFS curve of participants with advanced NSCLC treated with pembrolizumab $(n=24)$. PFS, progression-free survival; NSCLC, non-squamous cell lung cancer.

KRAS gene mutation was seen in 4 cases, 1 case showed a v-raf murine sarcoma viral oncogene homolog B1 (BRAF) gene mutation, and there were 19 cases without common driver gene mutations. Pembrolizumab monotherapy was received by 4 participants, and 20 received a combination treatment of pembrolizumab and chemotherapy. A total of 9 cases received first-line therapy or more before undertaking pembrolizumab therapy. The median period of pembrolizumab therapy was 7 (4-21 cycles).

\section{Efficacy assessment}

Follow-up information was available for all 24 participants, and the median follow-up time was 9 months (3-20 months). There were no deaths among these participants. A total of 11 participants achieved PR, 6 achieved SD, and 7 experienced PD. The ORR was $45.8 \%$ (11/24) and DCR was $70.8 \%(17 / 24)$. A total of 8 participants were referred for surgery after achieving PR, and 4 patients achieved pCR. The MPR was $75 \%(6 / 8)$ and pCR was $50 \%(4 / 8)$. The Kaplan-Meier curves for PFS are presented in Figure 1. The median OS and PFS were not reached due to the recency of this study.

\section{PD-L1 expression status}

Testing for PD-L1 was performed in 15 of 24 patients (Table 2). A total of 2 were PD-L1 negative, and both of them achieved SD after the 15-month follow-up and 5 -month follow-up, respectively, after pembrolizumab therapy. A total of 6 participants had high expression of PDL1 (TPS $\geq 50 \%$ ), but 1 of them had tumor progression after 
Table 2 Clinical Information of participants tested for PD-L1 ( $\mathrm{n}=15)$

\begin{tabular}{|c|c|c|c|c|c|c|c|c|c|c|c|}
\hline No. & Gender & Age & $\begin{array}{l}\text { Histology } \\
\text { type }\end{array}$ & $\begin{array}{l}\text { Clinical } \\
\text { stage }\end{array}$ & Driver mutation & Tissue & $\begin{array}{l}\text { PD-L1 } \\
\text { TPS }\end{array}$ & Antibody & $\begin{array}{c}\text { Efficacy } \\
\text { assessment }\end{array}$ & $\begin{array}{l}\text { Follow-up } \\
\text { (months) }\end{array}$ & $\begin{array}{c}\text { PFS } \\
\text { (months) }\end{array}$ \\
\hline 1 & $M$ & 61 & ADC & IV & BRAF & Lung & 0 & $22 \mathrm{C} 3$ & SD & 15 & - \\
\hline 2 & M & 51 & ADC & IV & KRAS & Lung & 10 & $22 \mathrm{C} 3$ & PR & 14 & - \\
\hline 3 & $M$ & 56 & SCC & IV & Pan-negative & Lung & 15 & $22 \mathrm{C} 3$ & PD & 13 & 5 \\
\hline 6 & $\mathrm{~F}$ & 56 & ADC & IIIB & Pan-negative & LN & 30 & $22 \mathrm{C} 3$ & PD & 9 & 6 \\
\hline 7 & $M$ & 68 & SCC & IIIA & Pan-negative & Lung & 30 & SP263 & PR & 9 & - \\
\hline 8 & M & 47 & LCLC & IV & Pan-negative & Lung & 70 & SP263 & PR & 8 & - \\
\hline 12 & M & 53 & LCLC & IIIA & Pan-negative & Lung & 30 & $22 \mathrm{C} 3$ & PR & 4 & - \\
\hline 13 & M & 72 & SCC & IIIA & Pan-negative & Lung & 60 & SP263 & PR & 4 & - \\
\hline 14 & M & 54 & SCC & IIIA & KRAS & Lung & 20 & $22 \mathrm{C} 3$ & PR & 4 & - \\
\hline 15 & M & 75 & ADC & IV & Pan-negative & Lung & 70 & $22 \mathrm{C} 3$ & SD & 3 & - \\
\hline
\end{tabular}

M, male; F, female; ADC, adenocarcinoma; SCC, squamous cell carcinoma; LCLC, large cell lung cancer; LN, lymph node; PR, partial response; SD, stable disease; PD, progressive disease; KRAS, Kirsten rat sarcoma viral oncogene homolog; BRAF, v-raf murine sarcoma viral oncogene homolog B1.

5 months of treatment with pembrolizumab.

\section{Safety assessment}

The recorded AEs (Table 3) were mainly grade $1-2$, and severe AEs (SAEs, grade $\geq 3$ ) were relatively rare; SAEs were observed in 4 patients, but there were no grade 5 AEs. The majority of grade 3-4 AEs were hematologic toxicity, including decreased white blood cells (12.5\%), decreased neutrophil count (12.5\%), and decreased platelet count (4.2\%). Grade 3 gastrointestinal symptoms were experienced by 2 participants (such as nausea, vomiting, or diarrhea). In addition, grade 3 hypothyroidism was shown in 2 cases (8.3\%). All participants with AEs were normalized with symptomatic treatment. No AEs resulted in the discontinuation of pembrolizumab therapy.

\section{Discussion}

In this study, we retrospectively reviewed 24 advanced NSCLC patients treated with pembrolizumab therapy in the Fujian Provincial Hospital between 2019 and 2020 and reported the efficacy and safety of pembrolizumab therapy.

The results of this study suggest that pembrolizumab has good efficacy in the treatment of advanced NSCLC. The median follow-up time was 9 months (3-20 months). There were no deaths during the follow-up period and both median OS and PFS were not reached. The ORR was $45.8 \%$ and DCR was $70.8 \%$, which was better than the results of previous KEYNOTE-189/407 Phase III clinical trials $(7,8)$. We considered the following possibilities: (I) the majority of participants $(91.7 \%)$ presented an ECOG score of $0-1$, which may be related to the proportion of participants who received pembrolizumab as a first-line therapy. Therefore, patients in better physical condition may receive a better curative effect from pembrolizumab therapy; (II) most participants tested for PD-L1 in our study were positive [13/15 (86.7\%)]. Previous studies showed that PD-L1 positive patients had better prognoses than those who were PD-L1 negative; (III) in the present study, $83.3 \%(20 / 24)$ of the participants were treated with combination pembrolizumab and chemotherapy. 
Table 3 Adverse events in 24 participants treated with pembrolizumab therapy

\begin{tabular}{lccc}
\hline Adverse events & Grade 0, $\mathrm{n}(\%)$ & All grades, $\mathrm{n}(\%)$ & Grade 3/4, $\mathrm{n}(\%)$ \\
\hline Hematologic adverse effect & $15(62.5)$ & $9(37.5)$ & $3(37.5)$ \\
White blood cell decreased & $15(62.5)$ & $5(20.8)$ & $3(12.5)$ \\
Neutrophil count decreased & $19(79.2)$ & $4(16.7)$ & $0(0)$ \\
Anemia & $20(83.3)$ & & $1(4.2)$ \\
Platelet count decreased & & $17(70.8)$ & $0(0)$ \\
Non-hematologic adverse effect & $7(29.2)$ & $8(33.3)$ & $0(0)$ \\
Gastrointestinal disorders & $16(66.7)$ & $2(8.3)$ & $0(0)$ \\
Skin disorders & $22(91.7)$ & $0(0)$ & $0(0)$ \\
Transaminases increased & $24(100.0)$ & $2(8.3)$ & \\
Creatinine increased & $22(91.7)$ & & \\
Hypoparathyroidism & & & \\
\hline
\end{tabular}

The combination of drugs showed a trend toward clinical benefit. However, the option of whether to employ the combination of drugs is also affected by previous treatments and the physical condition of patients. Overall, patients treated with pembrolizumab in our center showed good results, but longer periods of observation are required and larger samples are needed to confirm the efficacy and safety.

In the present study, 8 participants underwent surgery after 4-6 cycles of pembrolizumab treatment. A single participant was preoperatively diagnosed as clinical stage IV NSCLC with femur and ilium metastases by PETCT. After 6 cycles of pembrolizumab combined with chemotherapy, PET-CT reexamination demonstrated disappearance of bone metastasis and the clinical stage was downgraded to stage IIIA. Subsequently, the surgery was performed, and this case reached pCR. Postoperatively, no tumor relapse was observed during the 10 -month follow-up of this participant. Furthermore, 4 participants who were preoperatively diagnosed as clinical stage IIIA NSCLC and 3 with stage IIIB, respectively, achieved PR after pembrolizumab combined with chemotherapy preoperatively and underwent surgery subsequently. There was 1 case of conversion to thoracotomy in our cohort and other 7 cases were treated safely with single-utility port video-assisted thoracoscopic surgery. The MPR was $75 \%$ and pCR was $50 \%$. This suggests that pembrolizumab immunotherapy may provide an opportunity to receive surgery, as a local treatment, for patients with advanced NSCLC, and it is also a potential option for preoperative neoadjuvant therapy for NSCLC.

There were some limitations to this study. Not all participants were tested for PD-L1. The biomarker PDL1 must be tested in the case of metastatic NSCLC treated with pembrolizumab as first-line therapy, but it is not essential when pembrolizumab is used as a secondline treatment (13). The KEYNOTE-024/042 phase III clinical trials showed that pembrolizumab could significantly improve the survival of advanced NSCLC compared with chemotherapy irrespective of patient PDLI TPS $\geq 50 \%$ or PD-L1 TPS $\geq 1 \%$ status (14). However, the predictive value of PD-L1 for immunotherapy is still controversial. Patients with weakly positive PD-L1 and even PD-L1 negative may benefit from immunotherapy. The KEYNOTE-189/407 clinical trials $(7,8)$ revealed that neither PD-L1 nor tissue tumor mutational burden (TMB) could fully predict the therapeutic effect of anti-PD-1/ PD-L1 therapy. The expression of PD-L1 is regulated by two principle mechanisms. The first is the "endogenous" mechanism that tumor cell-intrinsic oncogene modulates PD-L1 expression via the intracellular signaling pathway. Second, the "exogenous" mechanism, is that the interferon $\gamma($ IFN- $\gamma)$ secreted by CD8+ T cells in the tumor immune microenvironment (TIME) induces PD-L1 expression on the surface of tumor cells (15). Thus, the effect of anti-PD-1/ PD-L1 therapy may also be affected by 
TIME, which is composed of stromal cells, immune cells, and immune-related molecules. The most convenient assessment of immune activity in TIME is through tumorinfiltrating lymphocytes (TIL) (16). Taube $e t$ al. proposed that the TIME be divided into 4 subtypes based on PDL1 and TIL: type I, PD-L1+/TIL high; type II, PD-L1-/ TIL low; type III, PD-L1+/TIL low; and type IV, PD-L1-/ TIL high (17). As for patients with type IV, tumor cells did not express PD-L1 or it was only poorly expressed, but significant quantities of TILs exist in tumor tissue, which suggests that the body has initiated an immunological reaction to the tumor and these patients will benefit from immunotherapy (18). Previous studies have also demonstrated that $\mathrm{PD}-\mathrm{L} 1$ positive patients had a high local response rate, because high PD-L1 expression was significantly associated with high TIL levels and high local response rate is relative to high TIL levels (19). As a result, recent studies have suggested that assessment of TIME based on multiplex immunohistochemistry (mIHC) has a higher accuracy in evaluating immunotherapy efficacy, as compared with PD-L1 and TMB (20). A total of two PDL1 negative patients were treated with pembrolizumab combined with chemotherapy and there was no significant progression during the follow-up period. This further confirms that PD-1/PD-L1 antibodies therapy has better performance of universality than targeted therapy. Targeted therapy is almost non-effective if there is no detected target for targeted therapy. The above results suggest that the predictive value of PD-L1 in immunotherapy for advanced NSCLC requires more detailed research, and there are still many unsolved mysteries for the exploration of biomarkers in immunotherapy.

For this study, pembrolizumab was given as a singleagent in two cases and the others were treated with a combination of pembrolizumab and platinum-containing chemotherapy. The overall incidence of AEs was $83.3 \%$ $(20 / 24)$ and the total incidence of SAEs (grade $\geq 3$ ) was $16.7 \%$, which is lower than that reported in previous clinical trials. The predominant AEs were grade 1-2 and no serious immune-related AEs (irAEs) were observed in this study. The SAEs (grade $\geq 3$ ) mainly included decreases in white blood cell and neutrophil counts, but the incidence of hematological SAEs was lower than that in previous studies on advanced NSCLC treated with platinum-based chemotherapy $(21,22)$. Overall, the observed AEs were consistent with those previously reported in clinical trials, which showed a low incidence of AEs and good tolerability of pembrolizumab in advanced NSCLC. Moreover, pembrolizumab may not increase the incidence of AEs when it is combined with chemotherapy, in comparison with the single chemotherapy.

\section{Conclusions}

In summary, pembrolizumab showed a good efficacy and low incidence of AEs for patients with advanced NSCLC. Pembrolizumab combined with chemotherapy can be used as the second or later-line therapy for patients with advanced NSCLC, and can also contribute an increased opportunity for curative surgical therapy for such patients. Therefore, pembrolizumab has broad prospects for application in the field of surgery.

\section{Acknowledgments}

Funding: None.

\section{Footnote}

Reporting Checklist: The authors have completed the STROBE reporting checklist. Available at http://dx.doi. org/10.21037/jtd-20-3413

Data Sharing Statement: Available at http://dx.doi. org/10.21037/jtd-20-3413

Conflicts of Interest: All authors have completed the ICMJE uniform disclosure form (available at http://dx.doi. org/10.21037/jtd-20-3413). The authors have no conflicts of interest to declare.

Ethical Statement: The authors are accountable for all aspects of the work in ensuring that questions related to the accuracy or integrity of any part of the work are appropriately investigated and resolved. This study was conducted in accordance with the declaration of Helsinki (as revise in 2013). The study was approved by the Institutional Research Ethics Board of the Fujian Provincial Hospital Ethics Committee (No.: 2020-015-01). Individual consent for this retrospective analysis was waived.

Open Access Statement: This is an Open Access article distributed in accordance with the Creative Commons Attribution-NonCommercial-NoDerivs 4.0 International 
License (CC BY-NC-ND 4.0), which permits the noncommercial replication and distribution of the article with the strict proviso that no changes or edits are made and the original work is properly cited (including links to both the formal publication through the relevant DOI and the license). See: https://creativecommons.org/licenses/by-nc-nd/4.0/.

\section{References}

1. Siegel RL, Miller KD, Jemal A. Cancer statistics, 2020. CA Cancer J Clin 2020;70:7-30.

2. Tartarone A, Lerose R, Aieta M. Focus on lung cancer screening. J Thorac Dis 2020;12:3815-20.

3. Lee J, Kefford R, Carlino M. PD-1 and PD-L1 inhibitors in melanoma treatment: past success, present application and future challenges. Immunotherapy 2016;8:733-46.

4. Reck M, Rodríguez-Abreu D, Robinson AG, et al. Pembrolizumab versus Chemotherapy for PD-L1Positive Non-Small-Cell Lung Cancer. N Engl J Med 2016;375:1823-33.

5. Le L, van Dams R, Lee P. Is first-line pembrolizumab appropriate for all patients with metastatic non-squamous histology non-small cell lung cancer patients? Transl Lung Cancer Res 2019;8:S327-S30.

6. Mok TSK, Wu YL, Kudaba I, et al. Pembrolizumab versus chemotherapy for previously untreated, PD-L1expressing, locally advanced or metastatic non-smallcell lung cancer (KEYNOTE-042): a randomised, open-label, controlled, phase 3 trial. Lancet 2019;393:1819-30.

7. Garassino MC, Gadgeel S, Esteban E, et al. Patientreported outcomes following pembrolizumab or placebo plus pemetrexed and platinum in patients with previously untreated, metastatic, non-squamous non-small-cell lung cancer (KEYNOTE-189):a multicentre, double-blind, randomised, placebo-controlled, phase 3 trial. Lancet Oncol 2020;21:387-97.

8. Paz-Ares L, Vicente D, Tafreshi A, et al. A Randomized, Placebo-Controlled Trial of Pembrolizumab Plus Chemotherapy in Patients With Metastatic Squamous NSCLC: Protocol-Specified Final Analysis of KEYNOTE-407. J Thorac Oncol 2020;15:1657-69.

9. AJCC Cancer Staging Manual, 8th ed, AJCC, New York 2017.

10. Lin G, Fan X, Zhu W, et al. Prognostic significance of
PD-L1 expression and tumor infiltrating lymphocyte in surgically resectable non-small cell lung cancer. Oncotarget 2017;8:83986-94.

11. Bohnsack O, Hooos A, Ludajic K. 1070P-Adaptation of the immune related response criteria: IRRECIST. Ann Oncol 2014;25:iv369.

12. National Cancer Institut, Common Terminology Criteria for Adverse Events, v 5.0, U.S. 2017 .

13. Ettinger DS, Wood DE, Aggarwal C, et al. NCCN Guidelines Insights: Non-Small Cell Lung Cancer, Version 1. 2020. J Natl Compr Canc Netw 2019;17:1464-72.

14. Nosaki K, Saka H, Hosomi Y, et al. Safety and efficacy of pembrolizumab monotherapy in elderly patients with PD-L1-positive advanced non-small-cell lung cancer: Pooled analysis from the KEYNOTE-010, KEYNOTE-024, and KEYNOTE-042 studies. Lung Cancer 2019;135:188-95.

15. Garcia-Diaz A, Shin DS, Moreno BH, et al. Interferon Receptor Signaling Pathways Regulating PD-L1 and PDL2 Expression. Cell Rep 2017;19:1189-201.

16. Pelekanou V, Carvajal-Hausdorf DE, Altan M, et al. Effect of neoadjuvant chemotherapy on tumor-infiltrating lymphocytes and PD-L1 expression in breast cancer and its clinical significance. Breast Cancer Res 2017;19:91.

17. Taube JM, Anders RA, Young GD, et al. Colocalization of inflammatory response with B7-h1 expression in human melanocytic lesions supports an adaptive resistance mechanism of immune escape. Sci Transl Med 2012;4:127ra37.

18. Shimizu K, Okita R, Saisho S, Maeda A, Nojima Y, Nakata $M$. Prognostic value of Cox-2 and PD-L1 expression and its relationship with tumor-infiltrating lymphocytes in resected lung adenocarcinoma. Cancer Manag Res 2017;9:741-50.

19. Schalper KA, Brown J, Carvajal-Hausdorf D, et al. Objective measurement and clinical significance of TILs in non-small cell lung cancer. J Natl Cancer Inst 2015;107:dju435.

20. Lu S, Stein JE, Rimm DL, et al. Comparison of Biomarker Modalities for Predicting Response to PD-1/PD-L1 Checkpoint Blockade: A Systematic Review and Metaanalysis. JAMA Oncol 2019;5:1195-204.

21. Satouchi M, Okamoto I, Sakai H, et al. Efficacy and safety of weekly nab-paclitaxel plus carboplatin in patients with advanced non-small cell lung cancer. Lung Cancer 
2013;81:97-101.

22. Hasegawa T, Futamura Y, Horiba A, et al. A phase II study of nab-paclitaxel plus carboplatin in combination with thoracic radiation in patients with locally advanced non- small-cell lung cancer. J Radiat Res 2016;57:50-4.

(English Language Editor: J. Jones)

Cite this article as: Guo T, Ding Y, Chen L, Zhu L, Lin J, Zhang J, Huang Y, Li W, Lin R, Pan X. Evaluation of pembrolizumab for the treatment of advanced non-small cell lung cancer: a retrospective, single-centre, single-arm study. J Thorac Dis 2021;13(1):282-290. doi: 10.21037/jtd-20-3413 